Voix et Images

voixetimages

\title{
Jacques Godbout, romancier
}

\section{Alain Piette}

Volume 11, numéro 1, automne 1985

\section{Naïm Kattan}

URI : https://id.erudit.org/iderudit/200544ar

DOI : https://doi.org/10.7202/200544ar

Aller au sommaire du numéro

\section{Éditeur(s)}

Université du Québec à Montréal

\section{ISSN}

0318-9201 (imprimé)

1705-933X (numérique)

Découvrir la revue

\section{Citer cet article}

Piette, A. (1985). Jacques Godbout, romancier. Voix et Images, 11(1), 119-122.

https://doi.org/10.7202/200544ar d'utilisation que vous pouvez consulter en ligne.

https://apropos.erudit.org/fr/usagers/politique-dutilisation/ 


\title{
Essai
}

\section{Jacques Godbout, romancier}

\author{
par Alain Piette, Université du Québec à Montréal
}

L'œuvre romanesque de Jacques Godbout a déjà été l'objet de nombreuses études, mais c'est la première fois qu'un ouvrage entier ${ }^{1}$ est consacré à l'aspect proprement narratif de l'ensemble des romans godboutiens. C'est sûrement là le premier mérite de ce travail où se révèle la grande familiarité de l'auteur avec son corpus - et c'est là le second mérite et non le moindre.

Le premier chapitre porté sur la trame événementielle. On peut s'interroger sur la nécessité de ce chapitre dans l'économie générale de l'ouvrage. Sans compter qu'il se réduit partiellement à des résumés des romans, il faut ajouter qu'il n'est pas très éclairant dans le cas de l'Aquarium par exemple (p. 16), roman dont l'histoire est déjà elle-même un peu confuse, et qu'il n'évite pas les redites (p. 17 et 19). On y relève de plus une imprécision de terme [cette première séquence, (p. 21), C'est comme un fond de scène qui permet aux autres séquences de se manifester, (p. 26)] où l'auteur semble avoir utilisé abusivement le terme séquence (pour intrigue ou thème?).

Le second chapitre se situe aussi au niveau de l'histoire racontée, mais du point de vue des personnages cette fois. En s'appuyant sur un témoignage de Jacques Godbout lui-même à propos de la seule Isle au dragon, l'auteur propose une grille de lecture adaptée à partir de Propp et Souriau. La description est menée de façon très minutieuse, peut-être trop dans le cas des protagonistes où une si longue démonstration était superflue pour prouver leur rôle de premier plan. Par ailleurs, il aurait fallu plus de vigilance pour départager chez les protagonistes les fonctions de l'acteur et celles du narrateur puisqu'il s'agit ici de narrateurs homodiégétiques (excluons pour le moment D'amour, P.Q. dont l'organisation narrative est plus complexe). Ainsi se trouve-t-on à lire des énoncés confus comme celui-ci: En écrivant leur journal, Charles et François livrent leur orientation. Ils conduisent l'action comme ils l'entendent, en faisant ressortir leurs tendances de même que leurs désirs (p. 49-50). La hiérarchie des niveaux narratifs a représenté une autre pierre d'achoppement pour l'auteur de l'essai. Cela l'amène à un contresens par exemple: L'opération qui a nécessité toute une panoplie d'instruments tranchants ressemble à une véritable boucherie (p. 69). Et l'auteur de citer un extrait du texte opératoire de François, texte fictif au second degré, comme s'il s'agissait de l'opération qui se produira dans la fíction au premier degré. De la même façon, on ne peut considérer Imroul ou Justman comme des adjuvants qui mettent en valeur les actants du récit premier ( $\mathrm{p}$. 77) puisque cela équivaut à fusionner deux structures narratives situées à des paliers différents. À cette erreur sur les niveaux narratifs s'ajoute une incompréhension de la notion d'adjuvant. Lorsqu'on lit: Quels personnages aident (je souligne) les narrateurs à résoudre leurs conflits? (p. 65), on a peine à reconnaître que l'énoncé vise les destinateurs et non les adjuvants. Le terme adjuvants mythiques de la page 87 laisse perplexe, surtout lorsque l'exemple à l'appui est l'allusion à Robespierre dans le discours du narrateur. Adjuvant ne peut plus signifier actant narratif dans 
un cas comme celui-là, pas plus que dans les allégories évoquées quelques lignes plus loin. L'analyse des fonctions actantielles ne garde son sens que si l'on maintient un plan homogène de lecture, littéral ou figuré, mais pas les deux à la fois.

Avec le troisième chapitre, on aborde le discours du récit dans le sens genettien. À ce propos, il aurait été préférable de citer explicitement Figures III, pour les définitions par exemple, plutôt que de le citer approximativement et sans guillemets. La première partie, sur l'ordre temporel, est très élaborée. Les analyses de micro-structures (p. 94 et suiv.) paraissent superflues. Chez Genette, ce développement jouait un rôle pédagogique qui n'a pas sa raison d'être ici. De plus, la classification en cinq catégories manque de pertinence. Toute micro-structure, à l'exception de la première et de la dernière d'un roman, est elle-même encadrée. On voit mal alors comment distinguer entre les deux ordres présent-passé et à passé-présent par exemple. Le reproche le plus grave qu'on pourrait adresser à cette troisième partie concerne - encore une fois - la notion de niveau narratif: Enfin, faut-il mentionner comme dernière catégorie d'analepses hétérodiégétiques les auires romans de $D$ 'amour, $P . Q$., si on considère comme récit premier le fait que Mireille écrit ou tape le roman de Thomas? (p. 104). L'analyse doit être menée à un même niveau, c'est-à-dire par rapport à la même trame événementielle. Ici, les romans dont l'essayiste parle sont des métarécits par rapport au récit premier. Certains types de prolepses repérés par l'auteur semblent aussi sujets à caution. L'établissement des anachronies en général suppose comme point de repère l'histoire racontée, donc la trame événementielle. Dans cette perspective, où situer les rêves comme celui de Galarneau (p. 92): sont-ils des métarécits, des discours de personnages? Le contenu des anticipations oniriques ou fantasmatiques n'appartient pas en tout cas à la trame événementielle du récit premier. Enfin, l'auteur, qui a bien décrit les anachronies dans l'ensemble, a parfois mal jaugé le caractère externe ou interne pour les analepses en particulier: ainsi l'évocation du meurtre de Vladimir (p. 103) n'est pas une analepse externe, mais en revanche, le rappel des nains Fontaine (p. 104), du passé de Monsignore (p. 105) et les réminiscences citées en page 107 constituent bel et bien des analepses externes. Externe aurait-il été entendu ici dans le sens d'étranger?

L'analyse de la durée narrative est plutôt réussie: des tableaux utiles sont présentés pour chaque roman et certaines remarques ne manquent pas de perspicacité comme c'est le cas du rapport établi entre l'absence de nom du narrateur et l'imprécision chronologique de sa biographie (p. 132). On note de petites erreurs révélant une incompréhension de l'expression récit premier (cela rejoint les erreurs sur les analepses externes et internes): ces deux romans commencent un printemps, l'un par la mort du père de François, l'autre par la naissance du bicéphale (p. 130). Je formulerais une dernière remarque: cette description aurait gagné à être complétée par des commentaires sur la vitesse du récit en rapport avec son contenu. Tous les matériaux y étaient: l'auteur a escamoté ici la partie interprétative en se repliant sur la seule description. 
Le quatrième chapitre réserve une surprise au lecteur: il annonce un développement sur le narrateur, mais il traite aussi du focalisateur. Le plan du chapitre forme d'ailleurs un tout hétéroclite: le statut du narrateur, le narrateur omniscient, le narrateur subjectif, le narrateur objectif, le narrateur-juge. Alors que les première et dernière parties concernent vraiment le narrateur, les trois autres visent le focalisateur malgré la présence du mot narrateur. On constate ici la confusion théorique de l'auteur à ce sujet, lequel reprend la division tripartite de Todorov et Genette mais en modifiant le terme médian au détriment de la clarté. Il convient de se demander pourquoi la théorie de Mieke Bal (qu'il cite) sur les instances narratives n'a pas été mise à contribution. Confusion théorique entre le sujet de l'énoncé et le sujet de l'énonciation, entre le personnage et le narrateur, confusion qui apparaît dans la partie «le statut du narrateur» à travers des développements parfois bizarres sur les marques pronominales. C'est au sujet de D'amour, P. Q. que se manifeste le plus nettement la mauvaise mise au point de l'essayiste (p. 149-150). Ce dernier n'a même pas entrevu que le narrateur du premier degré n'est pas Thomas D'Amour, mais un narrateur anonyme qui est le seulà pouvoir produire des énoncés attributifs comme dit Thomas $D^{\prime} A$ mour, dit Mireille. La partie sur le narrateur omniscient renferme parfois de fort bonnes observations, mais on souhaiterait que soient distinguées omniscience et intuitions (ou fantasmes) des narrateurs (p. 156). Le narrateur peut, à titre d'hypothèse, élucubrer sur ce que pense un personnage, ce qui n'assimile pas sa position à celle du narrateur d'Un homme et son péche par exemple faisant connaître les pensées intimes de Séraphin, Donalda et Alexis.

«Le plaisir du verbe», tel est le titre de cette dernière partie qui porte sur l'aspect plus strictement linguistique ou rhétorique du texte narratif. La division proposée en ce qui concerne les types de discours ne semble reposer sur aucun principe de cohérence: discours primitifs, connotatifs, énonciatifs et personnels. À côté de phénomènes d'ordre stylistique où l'auteur observe avec justesse des figures récurrentes comme l'ellipse et la parenthèse, il est beaucoup question dans ce chapitre de ce que Genette a appelé transtextualité dans son Palimpsestes ${ }^{2}$ : jeux intertextuels, architextualité (appartenance générique) et hypertextualité. Cette dernière catégorie est l'objet de développements particuliers, mais les définitions fournies pour la parodie et le pastiche laissent à désirer (p. 190). Lorsque Godbout par exemple emprunte la structure du journal intime, ce n'est pas que du pastiche. C'est ici en particulier que la notion d'hybridation bakhtinienne arriverait à point pour illustrer le nouveau régime textuel instauré par le mélange des genres (p. 191 et 195). Je dois enfin mentionner l'excellente partie sur le ton déclamatoire des romans de Godbout (p. 198-199). Il est déplorable toutefois qu'on n'y traite pas d'ironie, pas plus que dans le reste de cet essai. L'importance de ce procédé discursif dans ce corpus en fait un aspect essentiel de la stratégie narrative.

La dernière étude, sur la mise en abyme, manque vraiment de rigueur. Elle reflète une lecture superficielle de Dallenbach et propose souvent comme mises en abyme de simples figurations ou procédés iconiques où l'on n'observe même pas deux niveaux narratifs distincts. De plus, par un glis- 
sement abusif suggéré par l'exemple de Gide pour la peinture, le thème du double est développé à partir de la figure du miroir. On est loin de la division tripartite de Lucien Dällenbach!

En conclusion, il faut souligner une fois de plus l'immense travail accompli par Yvon Bellemare sur le corpus godboutien. Si j’ai dû émettre de fréquentes réserves, c'est à cause des nombreuses failles théoriques (en narratologie surtout) lesquelles entraînaient maintes applications fautives. On aurait pu souhaiter un élargissement de perspective, en conclusion surtout, une ouverture sur le social, lieu de prédilection de l'auteur, mais j'ai jugé plus pertinent de m'en tenir au projet même de l'essayiste.

1. Bellemare, Yvon, Jacques Godbout romancier, Montréal, Parti Pris, 1984, 242 pages.

2. Genette, Gérard, Palimpsestes, La littérature au second degré, Paris, Éditions du Seuil, coll. "Poétique», 1982, 468 pages. 